\title{
Jóvenes en Colombia y Brasil en una sociedad individualista en busca de trabajo digno y seguridad social*
}

\author{
Yaneth Vargas Sandoval** \\ Carla Rita Bracchi Silveira****
}

Recibido: 12 de octubre de 2018 - Aprobado: 18 de enero de 2019

\section{Resumen}

En la presente investigación se pretende hacer un análisis de la situación real en la que viven los jóvenes en América Latina, específicamente en Colombia y Brasil, señalando que son un bono demográfico que debe ser aprovechado en la región. La identificación de los jóvenes tan solo se hará en el siglo xx. En el marco legal de Brasil, como joven a las personas de 15 a 29 años y en Colombia a las de 14 a 28 años. Igualmente, se hará un acercamiento de los jóvenes y el trabajo, de los jóvenes y la transición educación-trabajo, de la informalidad en los jóvenes, y se

\footnotetext{
Este artículo es producto del proyecto de investigación institucional "La seguridad social en pensiones en Iberoamérica desde la perspectiva de género", gestionado en el marco del programa del Doctorado en Derecho en la Universidad Rey Juan Carlos, en España. Citar como: Vargas Sandoval, Y. y Bracchi Silveira, C. R. (2019). Jóvenes en Colombia y Brasil en una sociedad individualista en busca de trabajo digno y seguridad social. Revista IUSTA, 51(2), 115-134. DOI: https://doi. org/10.15332/25005286.5035

** Abogada por la Universidad Libre, magíster en Dirección y Gestión de los Sistemas de Seguridad Social por la Universidad Alcalá de Henares y magíster en Relaciones Internacionales Iberoamericanas por la Universidad Rey Juan Carlos. Docente de la Universidad Tecnológica de Cartagena y de la Universidad de Boyacá, Tunja, Colombia. Orcid: https://orcid.org/0000-00029188-1477. Correo electrónico: yvargas@uniboyaca.edu.co

*** Abogada por la Universidad Estatal de Santa Cruz, especialista en Derecho Ambiental por la Pontificia Universidad de Católica de Minas Gerais y magíster en Derecho del Trabajo y Relaciones Internacionales por la Universidad Nacional Tres de Febrero de Argentina. Docente de la Faculdad de Madre Thaís, Ilhéus, Brasil. Orcid: https://orcid.org/000-0002-7647-4267. Correo electrónico: carlabracchi@uol.com.br
} 
demostrará que esta situación afecta más a las mujeres que a los hombres. Sumado a lo anterior se evidencian los jóvenes que se encuentran excluidos de la sociedad, sin estudio y sin trabajo. Con esto se concluye que en Colombia y en Brasil no se hace una diferenciación del joven en el trabajo, ni en la seguridad social, lo que afecta de manera directa su acceso en un futuro a una pensión.

Palabras clave: juventud, trabajo formal, trabajo informal, seguridad social.

\title{
Young people in Colombia and Brazil in an INDIVIDUALISTIC SOCIETY IN SEARCH OF DECENT WORK AND SOCIAL SECURITY
}

\begin{abstract}
In this research, we intend to analyze the actual situation in which young people live in Latin America, specifically in Colombia and Brazil, noting that they are a demographic bonus that should be used in the region. The identification of young people will only be done in the twentieth century, defining in the legal framework of Brazil as young people those from 15 to 29 years and in Colombia those from 14 to 28 years. Likewise, there will be an approach of young people and work, of young people and the education-work transition, of informality in young people, demonstrating that this situation affects women more than men. In addition to the above, young people are found who are excluded from society, without study and without work. With this, it is concluded that in Colombia and Brazil there is no differentiation of the young people at work or in the social security, which directly affects their access to a pension in the future.
\end{abstract}

Keywords: youth, formal work, informal work, social security.

\section{Jovens na Colômbia e no Brasil em uma sociedade INDIVIDUALISTA EM PROCURA DE TRABALHO DIGNO E SEGURANÇA SOCIAL}

\section{Resumo}

Nesta pesquisa pretende-se fazer uma análise da situação real na qual vivem os jovens na América Latina, especificamente na Colômbia e no Brasil, assinalando 
que são um bônus demográfico que deve ser aproveitado na região. A identificação dos jovens somente se fará no século xx, definindo-se no marco legal do Brasil como jovens às pessoas de 15 aos 29 anos e na Colômbia as pessoas dos 14 aos 28 anos. Igualmente, se fará uma aproximação dos jovens e o trabalho, dos jovens e a transição educação-trabalho, da informalidade nos jovens, demonstrando que esta situação afeta mais às mulheres do que aos homens. Além do anterior evidencia-se aos jovens que estão excluídos da sociedade, sem estudo e sem trabalho. Com isso conclui-se que na Colômbia e no Brasil não se faz uma diferenciação do jovem no trabalho, nem na segurança social, o que afeta de maneira direta seu acesso no futuro a uma pensão.

Palavras-chave: juventude, trabalho formal, trabalho informal, seguridade social.

\section{Introducción}

Este trabajo tiene como objetivo principal hacer un análisis de la realidad laboral de los jóvenes de América Latina, específicamente de Colombia y Brasil. Por esta razón, en su primera parte, se evidencia cómo los jóvenes son la cuarta parte de la población en Colombia y Brasil, de modo que es un bono demográfico a tener en cuenta en la región. En la segunda parte, se plantea cómo ha sido el desarrollo del concepto de joven desde el punto de vista legal en Colombia y Brasil, que demuestra que solo a partir de la década de 1980 se hace un reconocimiento del concepto de joven en el desarrollo constitucional y legal.

En la tercera parte, se estudiará el trabajo y la juventud, y se demostrará que son pocos los jóvenes que pueden acceder a un trabajo en condiciones dignas, igualmente que las mujeres jóvenes son las que más sufren en un mayor porcentaje el desempleo. Asimismo, se analiza la transición educación-trabajo que enfrentan los jóvenes. En este sentido, tanto en Colombia como en Brasil, las normas que regulan los derechos de los jóvenes tendrán que incluir el componente de educación. Y si bien es cierto que tanto Colombia como Brasil tienen educación obligatoria, en Brasil hasta los 17 años y en Colombia hasta los 14 años, esta educación parece no estar cumpliendo con el objetivo de inserción de los jóvenes en el mercado laboral, lo cual se evidencia en los diez millones de jóvenes en América Latina que no consiguen emplearse (Organización Internacional del Trabajo [oIT], 2017, p. 42). 
En la cuarta parte, se analiza el desempleo y el trabajo infomal, planteándose que la no adecuada transición educación-trabajo de los jóvenes de familias pobres los obligan a enfrentar el círculo vicioso de la pobreza, ya que deben abandonar la educación a edades tempranas, y al no estar capacitados adecuadamente, el único camino laboral es el trabajo informal, lo que aumenta la inequidad social y la pobreza.

Igualmente, se hace un acercamiento a los jóvenes ninis, a los jóvenes que están excluidos de la sociedad, sin oportunidades y desesperanzados, de modo que es una realidad evidente en la región, principalmente para Colombia y Brasil.

$\mathrm{Y}$, por último, se analizará cómo los sistemas de seguridad social tanto en $\mathrm{Co}$ lombia como en Brasil no hacen una distinción a favor de ser joven, específicamente dentro de los sistemas de pensiones, ya que equiparan a todos como adultos; esta connotación hace que para los jóveves resulte muy difícil poder acceder al sistema pensional que desde sus principios está vinculado al empleo formal.

\section{Jóvenes}

Estudiar actualmente a los jóvenes es hablar de los que se encuentran inmersos en las nuevas tecnologías, en una sociedad basada en las redes y en la inmediatez de la posverdad. Pero también es estar en "una sociedad de consumidores cuyos proyectos de vida se construyen sobre las opciones de consumo y no sobre el trabajo" (Bauman, 2000, p. 11).

Del mismo modo, estos jóvenes están convencidos de que son seres individuales, que deberán enfrentar los riesgos solos. Según Castel (2010), se les hace creer que ellos deben solucionar sus propias realidades; el neoliberalismo los lleva a que se hagan una producción de ellos mismos frente al trabajo: "El yo como empresario de sí mismo se produce, se representa y se ofrece como mercancía” (Byung-Chul, 2018, p. 38).

Frente esta realidad, los jóvenes se deberán enfrentar con el trabajo, pero no cualquier trabajo, sino que será un trabajo en constante evolución, "que se traduce principalmente en la desocupación masiva y la precariedad de las relaciones de empleo" (Castel, 2010, p. 111), que los afecta de manera más directa.

La población joven de América Latina y el Caribe asciende a 140 millones de personas (Fondo de Población de las Naciones Unidas [FPNU], s. f.), por lo que podría señalarse que la región se encuentra con un bono demográfico a su favor. Pero es responsabilidad de los Estados aprovechar a su favor este bono, ya que con 
él se podrían mejorar aspectos económicos y sociales de la región para enfrentar adecuadamente la pobreza y la inequidad social (Cortés, 2016).

En Colombia, de acuerdo con el censo poblacional de 2018, elaborado por el Departamento Administrativo Nacional de Estadística (Dane), se determinó que la población colombiana asciende a 45,5 millones de personas. Y siendo una cuarta parte la población de jóvenes de edades entre 14 y 28 años, que ascienden a 12768 157, de los cuales 6518956 son hombres y 6249 201, mujeres.

Por su parte, Brasil, por ser el país más grande de América Latina, impacta los indicadores de la región en lo que respecta a la población, la cual asciende para 2017 a 207,7 millones de habitantes según el Instituto Brasileiro de Geografia e Estatística (Івge, 2018), entre ellos jóvenes de 15 a 24 años. Y porcentualmente el $16,43 \%$ del total de la población, que asciende a 17146060 hombres y a 16661 163 mujeres jóvenes.

En Brasil, la población considerada joven es de aproximadamente 51 millones de personas. Teniendo en cuenta que la población total del país gira en torno a 207 millones, los jóvenes representan igual que en Colombia la cuarta parte del total de la población (Mass Rocha, 2015).

\section{Ser joven en Colombia y Brasil dentro del marco legal}

Uno de los problemas que se plantean al analizar a los jóvenes, tanto en Colombia como en Brasil, es que dentro del marco histórico, específicamente para el siglo XIX, no es fácil su identificación como joven, debido a que en el rango determinado por edades solo se concebía a las personas como mayores y menores de edad, lo que hacía invisibles a los jóvenes dentro de la norma anterior a la década de 1980 (Saidiza, 2016).

En Colombia, se inicia la clasificación por edades de la población en la Constitución de 1886, que establece que serían mayores de edad las personas que tuviesen 21 años y menores a quienes tuviesen menos de dicha edad (Reina, 2012, p. 513). Esta mayoría de edad va a ser modificada a los 18 años en el Acto Legislativo 1/1975, del 18 de diciembre.

Es en la segunda mitad del siglo xx cuando se hace una verdadera identificación y reivindicación de los derechos de los jóvenes a nivel internacional, de modo que es la Organización de las Naciones Unidas (ONU) la que promueve y declara el Año Internacional de la Juventud en 1985. Colombia y Brasil comienzan en este periodo la identificación de los derechos de los jóvenes. 
Este reconocimiento de los jóvenes en Colombia se produce dentro de una sociedad que entra en la modernización con aspectos como "la democratización de la educación, el fortalecimiento del Estado de derecho, el afianzamiento de lo laico en la cultura, la mayor integración internacional y la expansión de los medios masivos de comunicación" (Sarmiento, 2000, p. 2).

Colombia en 1990 establece la Consejería Presidencial para la Juventud, la Mujer y la Familia, que se convertirá en 2000 en la Dirección del Sistema Nacional de Juventud "Colombia Joven”, dependencia del Departamento Administrativo de la Presidencia de la República.

En Brasil, será denominado Secretaría Nacional de Juventud (SNJ), creada en 2005, que igualmente tendrá como objetivo promover la investigación y el diagnóstico sobre la situación de la juventud brasileña, así como elaborar, consolidar y ejecutar las políticas nacionales que atiendan las demandas de los jóvenes.

Sumado a estas iniciativas, Colombia sufrirá una transformación como Estado con la expedición de la Constitución Política de 1991, en la cual se reconocen los derechos de los adolescentes, al mencionar que "tienen derecho a la protección y a la formación integral”. Este reconocimiento a nivel constitucional es un gran avance de los derechos de los jóvenes, que va a reforzarse al expedirse la Ley 375/1997, de 4 de julio que tendrá por objeto establecer y orientar las políticas y los programas por parte del Estado y la sociedad para la juventud.

Pero será mediante la Ley 1622/2013, de 29 de abril que se establecen lineamientos claros para la formulación de las políticas públicas de juventud y se crea el sistema de gestión del conocimiento, al identificar como joven a "toda persona entre 14 y 28 años cumplidos en proceso de consolidación de su autonomía intelectual, física, moral, económica, social y cultural que hace parte de una comunidad política y en ese sentido ejerce su ciudadanía”.

Respecto de la incorporación de los jóvenes al mercado laboral, en el Código Sustantivo del Trabajo expedido en 1950, se estableció la capacidad para celebrar contratos de trabajo a las personas mayores de 18 años (Decreto 2663/1950, de 5 de agosto). Pero, igualmente, se permite celebrar contratos de trabajo con menores de edad, siempre que tengan permiso de sus padres o del inspector del trabajo o primera autoridad local. En la Ley 1098/2006, de 8 de noviembre, se determinará que pueden trabajar los adolescentes de 15 o 16 años, siempre que laboren solo seis horas al día, y los jóvenes de 17 años 8 horas diarias, máximo en la semana 40 horas. 
Brasil, por su parte, presenta el mismo problema de identificar a los jóvenes dentro del marco legal. Solo hasta 2013 se promulga el Estatuto de la Juventud, Ley 12852/2013, de 5 de agosto por medio del cual se determinan cuáles son los derechos de los jóvenes que deben ser garantizados y promovidos por el Estado brasileño. La base del Estatuto de la Juventud es sustentada en principios, directrices y derechos, influenciados por la Constitución Política de la República Federativa de Brasil de 1988.

Según el Estatuto de la Juventud, se consideran jóvenes las personas de entre 15 años y 29 años (Ley 12852/2013, de 5 de agosto). El estatuto plantea igualmente en el artículo 14 el derecho de todo joven brasileño a la profesionalización, al trabajo en condiciones de "libertad, equidad y seguridad, adecuadamente remunerado y con protección social”.

En relación con la inclusión de los jóvenes en el mercado de trabajo, tanto el C138 - Convenio sobre la edad mínima, 1973 como la R146 - Recomendación sobre la edad mínima, 1973 sobre edad mínima para acceder a un empleo fueron aprobados por el Congreso Nacional de Brasil por medio del Decreto Legislativo 179/1999, de 14 de diciembre. De acuerdo con lo establecido en la Ley Orgánica 15/1999, de 19 de diciembre, aprobada por el Decreto 4.134/2002, de 15 de febrero. Por esta razón, a partir de este marco normativo, Brasil plantea la edad de 16 años como edad mínima para acceder a un empleo. En su artículo 3, se menciona:

Não será inferior a 18 (dezoito) anos a idade mínima para admissão a qualquer tipo de emprego ou trabalho que, por sua natureza ou circunstâncias em que for executado, possa prejudicar a saúde, a segurança e a moral do jovem. Entretanto a lei ou regulamentos nacionais ou a autoridade competente poderá, após consultar as organizações de empregadores e de trabalhadores concernentes, se as houver, autorizar emprego ou trabalho a partir da idade de 16 (dezesseis) anos, desde que estejam plenamente protegidas a saúde, a segurança e a moral dos jovens envolvidos e lhes seja proporcionada instrução ou formação adequada e específica no setor da atividade pertinente.

Es importante señalar que en la Constitución Política de la República Federativa de Brasil de 1988 se estableció la edad de 14 años como límite mínimo para cualquier trabajo, pero prohibió el trabajo a las personas menores de 16 años, excepto en condición de aprendiz, a partir de los 14 años. Esta observación en cuanto a la 
edad mínima tiene como objetivo alinear las relaciones de trabajo brasileñas a los designios de convenciones internacionales del trabajo; a ejemplo del C138 - Convenio sobre la edad mínima, 1973, trata evitar la explotación del trabajo infantil (Garzón, 2013).

De esta forma, el joven adolescente puede ocuparse como aprendiz en el grupo de edad entre 14 y 18 años en trabajos no clasificados como insalubres, peligrosos o penosos. También se debe respetar el tiempo limitado a los estudios del joven trabajador. Solo cuando cumpla 18 años, el trabajo del joven es regularizado como el de los trabajadores adultos. En cualquiera de estas condiciones, el trabajador joven goza de derechos laborales, así como los demás trabajadores en general.

Como se pudo ver en las normas de derecho laboral, no se hace ninguna diferenciación en cuanto a ser joven de más de 18 a 28 años y ser adulto. Esta condición de joven se desvanece dándoles trato a todos los jóvenes como mayor de edad, tanto en Colombia como en Brasil.

\section{Trabajo y juventud}

En lo que respecta a la vinculación laboral de los jóvenes en la formalidad, en Colombia la población joven ocupada de acuerdo con el Dane para el trimestre junio-agosto de 2018 se encuentra entre el 57,5\%, teniendo posibilidad de empleo más en los hombres con un 65,5\% que las mujeres que tan solo llegan al 49,2\%. Las actividades que más emplean jóvenes son las ramas de comercio, hoteles y restaurantes.

Igualmente, se presenta una tasa de desempleo del 16,4\%, medición que evidencia la posición en desventaja en que se encuentran las mujeres jóvenes quienes soportan un desempleo del $21,4 \%$, en tanto que los hombres se encuentran desempleados tan solo el 12,7 \% (Dane, 2018).

En Brasil, oficialmente el IBGE no determina cuántos jóvenes de 15 a 29 años se encuentran en el mercado laboral, debido a que como se evidenció persiste el concepto de asimilar los jóvenes a los adultos, sin ninguna diferenciación.

Pero, tanto en Colombia como en Brasil para aprovechar el potencial de capital humano de jóvenes que se tiene, se hace necesario que accedan a condiciones de trabajo dignas. Sin embargo, la situación que viven actualmente los jóvenes es compleja, debido a que deberán enfrentar desafíos como la incorporación en los sistemas educativos y luego hacer la transición al mercado laboral, ya que solo si los 
jóvenes tienen una adecuada educación podrán acceder a empleos en condiciones dignas, mientras que los que no acceden a la educación o la abandonan a una edad temprana tendrán que enfrentarse al trabajo precario.

\section{Jóvenes en la transición educación-trabajo}

Un aspecto importante en la incorporación de los jóvenes al mercado laboral es la educación, por lo que en Colombia como en Brasil, dentro de las normas que regulan a los jóvenes, un aspecto que se tendrá en cuenta será la educación.

En el Estatuto de la Juventud de Brasil en el artículo 7, se establece el derecho de los jóvenes brasileños a acceder a la educación de calidad, de modo que la educación básica es de carácter obligatorio. La norma asimismo amplía claramente la educación a los jóvenes del campo, a los negros y a los indígenas, educación que debe otorgarse en niveles profesional, técnica o tecnológica. Con ello se pretende hacer una adecuada transición de los jóvenes al mercado laboral. El Estatuto, dentro del contexto de trabajo decente, señala en el artículo 14 que "o jovem tem direito à profissionalização, ao trabalho e à renda, exercido em condições de liberdade, equidade e segurança, adequadamente remunerado e com proteção social".

Colombia lo planteará en forma similar en la Ley 1622/2013, de 29 de abril, por medio de la cual se expide el estatuto de ciudadanía juvenil y se dictan otras disposiciones, marco legal que establece en el artículo 8 en las medidas de promoción que se debe garantizar a los jóvenes en Colombia una educación de calidad, incentivando que ellos permanezcan en la educación básica, secundaria, técnica y universitaria. Con lo que Colombia también pretende promover en los jóvenes "un empleo y unas condiciones de trabajo de calidad, y potenciar mecanismos de orientación e inserción laborales".

La educación no está dando la posibilidad de una adecuada inserción laboral

Tanto Colombia como Brasil, en los últimos años, han bajado las tasas de analfabetismo, debido a la implementación en el sistema educativo de la obligatoriedad de la educación básica y parte del nivel secundario, lo que conlleva que los jóvenes permanezcan en el sistema educativo hasta los 17 años en Brasil y hasta los 14 años en Colombia (Organización de las Naciones Unidas para la Educación, la Ciencia y la Cultura [Unesco], 2016). 
Esta educación parece que no está cumpliendo con el objetivo de una adecuada inserción en el mercado laboral, ya que en América Latina más de diez millones de jóvenes no consiguen emplearse (oit, 2017, p. 42). Para los jóvenes, tener el título de bachiller en Colombia como en Brasil ya no es una garantía para acceder a un trabajo digno. Beck establece que parece que cuando sale del sistema educativo actualmente el joven "queda degradado a una 'no formación' que históricamente casi se equipara al analfabetismo [las cursivas son mías] [...] para los niveles formativos inferiores (escuela primaria y escuela de educación especial)" (Beck, 1998, p. 194).

Pero para los jóvenes que llegan a un nivel de educación profesional la situación también es desesperanzadora, ya que igualmente a estos jóvenes les cuesta acceder a empleos de calidad, por lo que, amenazados por el desempleo, aceptarán un primer empleo en condiciones precarias, para no quedar marginados del sistema ocupacional (Beck, 1998, pp. 190 y 191). Lo anterior evidencia que el sistema educativo parece no estar cumpliendo con las expectativas de los jóvenes: terminar los estudios no asegura un futuro mejor, pero no tenerlos los somete a condiciones precarias.

\section{Jóvenes: desempleo y trabajo informal}

Para la oit (2017), los jóvenes son los que más sufren el desempleo en la región. "La tasa de desempleo juvenil promedio subió de 18,9 por ciento a 19,5 por ciento en 2017, lo cual significa que uno de cada cinco jóvenes en la fuerza laboral no consigue trabajo. Se estima que esta situación afecta a unos 10 millones de jóvenes" (p. 42). De acuerdo con la oiT, para 2018, Brasil y Colombia son los países que más se ven afectados por esta situación en la región.

Pero son los jóvenes de bajos recursos, de áreas rurales, que han dejado los estudios, muchas veces para el sostenimiento de sus familias, quienes deben laborar a más temprana edad (Unesco, 2016). Sin una educación por lo menos básica, las posibilidades de empleos de calidad se reducen considerablemente. Esta inadecuada transción entre escuela-trabajo tendrá como consecuencia que los jóvenes deban trabajar en el sector informal como único camino viable dentro del mercado laboral (Organización para la Cooperación y el Desarrollo Económicos [ocDE], 2016).

El trabajo informal entonces para estos jóvenes se transforma en un ciclo vicioso de pobreza e inequidad social. Estas altas tasas de informalidad laboral en América Latina y el Caribe plantean grandes "desafíos socioeconómicos en la región" (OCDE, 2018). 
En Colombia, los jóvenes son los que más se emplean en trabajos informales (ort, 2015). En el trimestre de julio-septiembre de 2018, la informalidad asciende al 48,0 \% (Dane, 2018). Los jóvenes colombianos, al no poder acceder a un trabajo digno, afectan de manera directa la seguridad social, sobre todo en pensiones, lo que generará en un futuro que estos jóvenes no tengan el derecho a un reconocimiento pensional específicamente en la pensión de vejez.

Por su parte, Brasil es responsable del $80 \%$ de la población joven del Cono Sur y del $50 \%$ de los jóvenes de América Latina. Por un lado, estas cifras representan una gran capacidad de crecimiento para el país; por otro, no hay suficiente estructura para proporcionar salud, educación y empleo para todos estos jóvenes a corto o a medio plazo.

Los estudios de la ONU y del IBGE confirman que nunca hubo y nunca más habrá en el país una densidad demográfica tan grande de jóvenes. Estos, que están en el grupo de edad de 15 a 24 años, necesitan ser atendidos en sus necesidades más básicas y ser orientados a asumir el nuevo Brasil.

La peculiaridad de la juventud brasileña revela su heterogeneidad, desigualdades y exclusión social. Según la oit (2009), "las mujeres jóvenes, los jóvenes negros de ambos sexos, así como los jóvenes de las áreas metropolitanas de bajos ingresos, o de determinadas zonas rurales, son afectados de forma más severa por la exclusión social, la falta de oportunidades y el déficit de empleo de calidad".

Para los jóvenes brasileños, el acceso al empleo y la educación escolar de calidad y la exposición a la violencia pueden ser considerados los grandes desafíos de la actualidad. A pesar de esas dificultades enfrentadas en esas tres dimensiones, no son exclusividad de la juventud; es para esa categoría social que ellos se profundizan $\mathrm{y}$ tienen efectos directos sobre su futuro.

Además, los recortes en programas sociales como el Programa Universidad para Todos (ProUni) y el Fondo de Financiamiento Estudiantil (FIEs) dificultan el acceso a los jóvenes a la enseñanza superior y, consecuentemente, la posibilidad de una formación diferenciada para el mercado de trabajo. De esta forma, los jóvenes buscan salidas en la informalidad — que alcanza el $38 \%$ en esa misma franja etaria - realizando actividades tan solo de supervivencia del día a día. 


\section{Jóvenes excluidos de la sociedad, ninis}

Sumado a la difícil situación que viven los jóvenes, en la sociedad actual, existen 30 millones en América Latina que no están incluidos en los lineamientos económicos ni sociales, por lo que se encuentran excluidos socialmente. Lo anterior se presenta porque ni estudian ni trabajan, parece que lo único que hacen es vivir en la desesperanza, sin futuro y culpabilizados de su propia realidad.

Culpa que se basa en la "sociedad de los individuos" en la que les ha correspondido vivir, en la que lo fundamental es la individualidad y en la que cada individuo se hace responsable de su propia existencia (Castel, 2010, p. 200). Pero no es una opción de los jóvenes estar excluido, sino que es es una política neoliberal la que los lleva a no tener oportunidades (Huertas, 2016).

"Uno de cada cinco jóvenes de América Latina no tiene empleo, no estudia ni recibe capacitación", esta es una realidad con contexto de género, son las mujeres jóvenes que llegan al $30 \%$ de las mujeres que no estudian, no trabajan y no reciben capacitación, frente al 11 \% de los hombres (ocDE, 2016, p. 28). Muchas de estas mujeres jóvenes realizan actividades de cuidadoras, actividades que no son remuneradas y menos consideradas como trabajo.

En América Latina, las cifras de ninis más altas se encuentran en Brasil y Colombia (De Hoyos, Rogers y Székely, 2016, p. 11). En Colombia, uno de los factores determinantes es la inequidad en la que los jóvenes se encuentran inmersos. La mayoría de los ninis provienenen de hogares pobres, en los que los jóvenes forman hogares a muy temprana edad, sumado a los embarazos de niñas y adolescentes, deserción escolar, asumen roles que no les corresponde, como lo es la ayuda a las necesidades básicas del vínculo familiar (Ochoa, Silva y Sarmiento, 2015, pp. 149-162).

En Colombia, debido a la realidad social de violencia, narcotráfico y conflicto armado en la que viven los jóvenes, la situación de exclusión social, y de no tener posibilidades de estudiar o de trabajar, los lleva a formar parte en muchos casos del crimen organizado, lo que aumenta su vulnerabilidad y los deja sin futuro (Hoyos et al., 2016, p. 1).

El fenómeno en Brasil de los nem-nem es igualmente preocupante: son 11 millones de jóvenes en esta situación; esta exclusión social va en aumento: para 2014, el porcentaje fue del 22,7 \% y para 2018 ya asciende a un 25,8 \%, según la reciente encuesta divulgada por el IBGE (2018). 
Las dificultades cotidianas impuestas por las diferencias regionales (el norte y noreste tienen los mayores índices de desempleados), género, grado de escolaridad y situación económica, son algunas de las razones que permiten el surgimiento de los nem-nem en Brasil (Vargas y Bracchi, 2016).

Es importante destacar que factores como el embarazo precoz, la ausencia de transporte público y seguridad en varias localidades y la enseñanza pública escasa son significativas barreras que obstaculizan la salida de los jóvenes de la condición de nem-nem en Brasil.

Esta población es una mano de obra que pronto envejecerá, por lo que se requieren soluciones inmediatas a estos jóvenes excluidos que tiene Brasil (Banco Mundial, 2018).

\section{Jóvenes y seguridad social}

Como se ha evidenciado hasta este momento en el presente artículo, los jóvenes en América Latina, y específicamente en Colombia y Brasil, se encuentran enfrentando una verdadera crisis desde el punto de vista laboral, una inadecuada transición entre educación-trabajo, un alto índice de informalidad y trabajos precarios. O no ser siquiera tenidos en cuenta por la sociedad, al ser declarados excluidos y solo ser considerados como ninis.

Todas estas situaciones afectan de manera directa el sistema de seguridad social, que hará que en un futuro no puedan acceder al sistema de pensiones, específicamente la pensión de vejez, porque desde sus orígenes la seguridad social en pensiones está ligada al trabajo formal.

Los jóvenes deben enfrentar entonces una sociedad contemporánea del riesgo, como lo plantea Castel (2010, p. 30), "vivimos tiempos inciertos", en una sociedad del riesgo, en la que además surgen nuevos como "el riesgo desocupación y el riesgo precariedad, que, si bien tampoco son nuevos, adquirieron tal amplitud que desestabilizan en profundidad nuestro sistema de protección social” (p. 31).

$\mathrm{Y}$ aun cuando los jóvenes cuentan con una norma específica para ellos que es la Ley 1622/2013, de 29 de abril, en lo que respecta al sistema pensional, son equiparados a los mayores de edad, sin ninguna diferenciación.

$\mathrm{Y}$ en Colombia, como todo ciudadano mayor de edad, en este caso asimilan igualmente a los jóvenes, quienes vinculados al trabajo formal deberán hacer aportes de manera obligatoria al sistema pensional. Siendo dual el sistema, pero excluyente 
entre sí, o se cotiza al régimen solidario de prima media con prestación definida, administrada por Colpensiones, fundamentado en la solidaridad intergeneracional, o las cotizaciones deben hacerse al régimen de ahorro individual con solidaridad administrado por las administradoras de fondos privadas de pensiones, fundamentado en el ahorro individual.

Actualmente, los jóvenes se encuentran en su mayoría afiliados a las administradoras privadas de fondos de pensiones con 2710405 afiliados, de los cuales solo se encuentran cotizando en pensiones 1890295 (R146 - Recomendación sobre la edad mínima, 1973). La cifra de los jóvenes que hacen cotizaciones sobre uno a tres salarios mínimos mensuales legales vigentes (SMMLV) está en 1794143 y mayor a cuatro smmLv es de 96135 afiliados cotizantes. Por esta razón, posiblemente se pensionen con una pensión de salario mínimo quienes cotizan arriba de tres SMmLV, los de menos tendrán que ser financiados con garantía de pensión mínima, esto es, ayudados por el Estado para acceder a una pensión con salario mínimo. Esto evidencia la dificultad que tienen los jóvenes colombianos en acceder a una adecuada prestación de seguridad social, y específicamente a una pensión de vejez.

En Brasil, la afiliación en el Regime Geral da Previdência Social se da mediante la inscripción en el Instituto Nacional de Seguro Social (INss) conforme determina el Decreto 3.048/1999, de 6 de mayo de 1999. El Regime Geral da Previdência Social no hace distinción entre jóvenes (15 a 29 años) y adultos (mayores de 29 años) para la inscripción y recepción de beneficios. El joven será un contribuyente obligatorio o facultativo según los términos del artículo 12 de la Ley 8212/1991, de 24 de julio, actualizada por la Ley 10.256/2001, de 9 de julio y el artículo 11 de la Ley 8.213/1991, de 24 de julio. Como en Brasil no hay determinación legal previsional específica para jóvenes, para que el joven pueda gozar del beneficio deberá contribuir con una cuota determinada.

Todo lo anterior evidencia que la sociedad actual, en la que les ha correspondido vivir a los jóvenes, es una sociedad en la que lo fundamental es la individualidad, que obliga a los individuos a ser todos iguales y autónomos, aun cuando no tengan recursos o las suficientes capacidades para desarrollarse como los demás en la sociedad. Tal vez por eso se les vendió la idea de un sistema pensional individual en el que ellos solos podrían ganar su propia pensión (Ballesteros, 2015).

Pero como hemos visto, la situación laboral de los jóvenes en América Latina, y específicamente en Colombia y Brasil, no es una realidad próspera y enfrentar a 
los jóvenes al individualismo parece no ser la mejor de las fórmulas. Para aquellos que no tienen oportunidades

la exhortación en ser un individuo se ha convertido en nuestra sociedad en un imperativo categórico sustentado por la ideología liberal dominante: ser autónomo y responsable, demostrar iniciativa, asumir uno mismo los riesgos. Pero, según esta lógica, se hace pesar sobre todos los individuos, incluso sobre aquellos que no tienen los recursos objetivos para enfrentarla, esa exigencia de conducirse como sujetos independientes. (Castel, 2010, pp. 205-206)

\section{Conclusiones}

A modo de conclusión podríamos manifestar que sin duda los jóvenes son la cuarta parte de la población no solo en Colombia y Brasil, sino en América Latina. E1 concepto de joven y la identificación como tal solo se va a dar en el siglo xx implementando su identificación y derechos en la Constitución de 1991 de Colombia y en la Constitución Política de la República Federativa de Brasil de 1988; ambos países para reivindicar los derechos de los jóvenes van a hacer estatutos de jóvenes a fin de desarrollar de una manera más amplia sus derechos.

Pero ser joven, desde el punto de vista laboral, no se diferencia en nada, ni tendrá beneficios en la realidad laboral que deben enfrentar. Sumado a que como son jóvenes deberán hacer la transición educación-trabajo. Y la educación, que se plantea como un elemento fundamental para obtener trabajos dignos para los jóvenes, no está cumpliendo con este objetivo, de modo que es deficiente en otorgar herramientas a estos en el mundo laboral.

Por otro lado, se puede concluir que ser pobre, mujer y sin educación agrava aún más la precaria condición de empleabilidad de los jóvenes, quienes en estas circunstancias enfrentan de manera directa la informalidad y los trabajos precarios.

Pero, igualmente, se evidencia cómo en Brasil y Colombia se tiene la población más alta de jóvenes que son excluidos de la sociedad al no poder tener acceso al trabajo, al estudio o a la capacitación. Excluidos en una sociedad que premia el individualismo, son castigados al hacérseles responsables de su propia situación. 
Y, por último, se puede concluir que sin trabajo digno muy difícilmente los jóvenes podrán acceder a un sistema de seguridad social que les garantice en un futuro una pensión.

\section{Referencias}

Acto Legislativo 1/1975, de 18 de diciembre, por el cual se modifican los artículos 14, 15 y 171 de la Constitución Nacional.

Ballesteros Moreno, M. C. (2015). La mujer y el ejercicio efectivo del poder en Colombia: una cuestión de derechos humanos. Verba Iuris, 33, 61-76.

Banco Mundial (2018, marzo 17). Jóvenes que no estudian ni trabajan: jelección o falta de opciones? Recuperado de https://www.bancomundial.org/es/news/feature/2018/03/17/ brasil-estudio-jovenes-no-estudian-ni-trabajan-ninis-genero-pobreza

Bauman, Z. (2000). Trabajo, consumismo y nuevos pobres. Barcelona, España: Gedisa.

Beck, U. (2002). La sociedad del riesgo: hacia una nueva modernidad. Buenos Aires, Argentina: Paidós.

Byung-Chul, H. (2018). La expulsión de lo distinto (A. Ciria, trad.). Barcelona, España: Herder.

C138 - Convenio sobre la edad mínima, 1973 (núm. 138) Convenio sobre la edad mínima de admisión al empleo (Entrada en vigor: 19 junio 1976).

Castel, R. (2010). El ascenso de las incertidumbres: trabajo, protecciones, estatuto del individuo. Buenos Aires, Argentina: Fondo de Cultura Económica.

Conferencia Internacional del Trabajo, 101. ${ }^{a}$ reunión, 2012. Recuperado de https://www. ilo.org/public/libdoc/ilo/2012/112B09_39_span.pdf

Constitución Política 1991, de 13 de junio, Constitución Política de 1991.

Constitución Política de la República Federativa de Brasil 1988. 
Cortés Zambrano, S. P. (2016). Derechos humanos en las políticas de paz y posconflicto en Colombia. Via Inveniendi et Iudicandi, 11(1), 129-145. DOI: https://doi.org/10.15332/ s1909-0528.2016.0001.03

Decreto 2663/1950, de 5 de agosto, Código Sustantivo del Trabajo Adoptado por el Decreto Ley 2663 del 5 de agosto de 1950 "Sobre Código Sustantivo del Trabajo", en virtud del Estado de Sitio promulgado por el Decreto Extraordinario No 3518 de 1949.

Decreto 3.048/1999, de 6 de mayo de 1999, aprova o regulamento da previdência social, e dá outras providências.

Decreto 4.134/2002, de 15 de febrero, Promulga a Convenção no 138 e a Recomendação no 146 da Organização Internacional do Trabalho (OIT) sobre Idade Mínima de Admissão ao Emprego.

Decreto Legislativo 179/1999, de 14 de diciembre, por el que se aprueban los textos del Convenio (núm. 138) y de la Recomendación (núm. 146) sobre la edad mínima, 1973.

Departamento Administrativo Nacional de Estadística [Dane]. (2018, octubre 10). Boletín técnico. Recuperado de https://www.dane.gov.co/files/investigaciones/boletines/ech/ juventud/Bol_eje_juventud_jun18_ago18.pdf

Departamento Administrativo Nacional de Estadística [Dane]. (2019, febrero 12). Boletín técnico. Recuperado de https://www.dane.gov.co/files/investigaciones/boletines/ech/ juventud/Bol_eje_juventud_oct18_dic18.pdf

Fondo de Población de las Naciones Unidas (s. f.). Adolescencia y juventud. Recuperado de https://lac.unfpa.org/es/temas/adolescencia-y-juventud

Garzón Buenaventura, E. F. (2013). Globalización del derecho, fetichismo legal: el velo de los derechos humanos. Verba Iuris, 30, 169-181.

Hoyos, R. de, Rogers, H. y Székely, M. (2016). Ninis en América Latina: 20 millones de jóvenes en busca de oportunidades. Washington, D. C., EE. UU.: Banco Mundial. 
Huertas Díaz, O. (2016). Entre la minimización y la expansión del derecho penal: la presencia de Beccaria en el debate contemporáneo. IUSTA, 1(44), 41-59. DOI: https:// doi.org/10.15332/s1900-0448.2016.0044.02

Instituto Brasileiro de Geografía e Estadística (2018). Estadísticas población brasilera. São Paulo, Brasil: Autor.

Ley 8212/1991, de 24 de julio, actualizada por la ley 10.256 del 9 de julio de 2001, por la que se dispone sobre la organización de la seguridad social, se instituye un plan de gastos, y se dictan otras disposiciones.

Ley 8.213/1991, de 24 de julio, actualizada al 27 de diciembre del 2000, por la que se dispone sobre los planes de beneficios de la previsión social, y se dictan otras disposiciones.

Ley 100/1993, de 23 de diciembre, por la cual se crea el sistema de seguridad social integral y se dictan otras disposiciones.

Ley 375/1997, de 4 de julio, por la cual se crea la ley de la juventud y se dictan otras disposiciones.

Ley Orgánica 15/1999, de 19 de diciembre.

Lei n. ${ }^{\circ} 12.852$ 2013, de 5 de agosto, Estatuto da Juventude. Establecer los derechos de los jóvenes y las directrices de las políticas públicas de juventud y el Sistema Nacional de la Juventud (sinajuve).

Ley 1622/2013, de 29 de abril, por medio de la cual se expide el estatuto de ciudadanía juvenil y se dictan otras disposiciones.

Ley 1098/2006, de 8 de noviembre, por la cual se expide el Código de la Infancia y la Adolescencia.

Mas Rocha Paes, F. D. (2015). Reflexiones acerca de la efectividad de la ley de tortura en Brasil: la importancia de la fase preprocesal. IUSTA, 1(42), 117-128. DOI: https://doi. org/10.15332/s1900-0448.2015.0042.02 
Ochoa Díaz, D., Silva Arias, A. C. y Sarmiento Espinel, J. A. (2015). Actividades y uso del tiempo de las y los jóvenes que ni estudian ni trabajan en Colombia. Civilizar Ciencias Sociales y Humanas, 15(29), 149-162.

Organización para la Cooperación y el Desarrollo Económicos (2016). Perspectivas económicas de América Latina 2017: juventud, competencias y emprendimiento. París, Francia: Autor.

Organización para la Cooperación y el Desarrollo Económicos (2018). Perspectivas económicas de América Latina 2018: repensando las instituciones para el desarrollo. París, Francia: Autor.

Organización para la Cooperación y el Desarrollo Económicos (2009). Trabajo decente y juventud en Brasil. Lima, Perú: Autor.

Organización Internacional del Trabajo [orT] (2015). Formalizando la informalidad juvenil: experiencias innovadoras en AméricaLatina y el Caribe. Lima, Perú: Autor.

Organización Internacional del Trabajo [оIт] (2017). Panorama Laboral 2017: América Latina y el Caribe. Lima, Perú: Autor.

Organización Internacional del Trabajo [оіт] (2017, diciembre 18). En 2017 el desempleo aumentó por tercer año consecutivo, pero bajaría el próximo año. Recuperado de https://www. ilo.org/global/about-the-ilo/newsroom/news/WCMS_614167/lang--es/index.htm

Organización de las Naciones Unidas para la Educación, la Ciencia y la Cultura [Unesco]. (2016). El nivel de deserción en Ibeoroamerica. Autor.

Presidencia de la República de Colombia (s. f.). Reportes vigentes. Recuperado de http:// obs.colombiajoven.gov.co/Observatorio/ObservatorioInicio.aspx

R146 - Recomendación sobre la edad mínima, 1973 (núm. 146) Recomendación sobre la edad mínima de admisión al empleo.

Registraduría Nacional del Estado Civil (2016, agosto 2). Historia del voto en Colombia. Recuperado de https://www.registraduria.gov.co/-Historia-del-voto-en-Colombia-.html

Reina Rodríguez, C. A. (2012). Historia de los jóvenes en Colombia 1903-1991 (Tesis doctoral, Universidad Nacional de Colombia, Bogotá, Colombia). 
Saidiza, V.H. (2016). Crisis del Estado de derecho en Colombia: un análisis desde la perspectiva de la legislación penal. IUSTA, 1(44), 17-39. DOI: https://doi.org/10.15332/ s1900-0448.2016.0044.01

Sarmiento Anzola, L. (2000). Política pública de juventud en Colombia: logros, dificultades y perspectivas. Bogotá, Colombia: Programa Presidencial Colombia Joven. Recuperado de http://207.58.191.15:8180/xmlui/handle/123456789/100

Sistema de Información de Tendencias Educativas en América Latina. (s. f.). El trabajo de mercado como obstáculo a la escolarización de los adolescentes. Recuperado de http://www.publicaciones.siteal.iipe.unesco.org/eventos-del-siteal/25/ el-trabajo-de-mercado-como-obstaculo-la-escolarizacion-de-los-adolescentes

Vargas Sandoval, Y. (2016). La mujer latinoamericana dentro del contexto de los derechos humanos: énfasis desde la perspectiva del derecho al trabajo. IUSTA, 1(44), 75-97. DOI: https://doi.org/10.15332/s1900-0448.2016.0044.04 